\title{
Traitement des données topographiques et bathymétriques acquises sur le littoral aquitain : optimisation des mesures effectuées en quad et au théodolite
}

\author{
Jean-Paul PARISOT ${ }^{1}$, Sylvain CAPO ${ }^{1}$, Stéphane BUJAN ${ }^{1}$, \\ Nadia SENECHAL ${ }^{1}$ et Jean BRILLET ${ }^{2}$ \\ ${ }^{1}$ Equipe METHYS. UMR 5805-EPOC/OASU, Université Bordeaux 1. Avenue des Facultés, \\ 33405, Talence, France et ANR VULSACO. \\ parisot@epoc.u-bordeaux1.fr \\ ${ }^{2}$ Laboratoire d'Astrophysique de Bordeaux .UMR 5804-LAB/OASU. 2, Rue de l'Observatoire, BP \\ 89, 33270, Floirac, France \\ brillet@observ.u-bordeaul.fr
}

\section{Résumé :}

L'équipe de recherche METHYS effectue régulièrement des levés topographiques et bathymétriques sur le littoral Aquitain. Ces mesures effectuées en quad, sont complétées par des mesures à pied ; des compléments de bathymétrie peuvent-être effectués au théodolite ou en scooter de mer. Afin de préserver une précision centimétrique sur tous les levés, une analyse soignée a été menée afin d'optimiser les différents stades du traitement (grille et fréquence des levés, grille de calcul, méthodes d'interpolation...). En particulier, des tests ont permis d'optimiser l'utilisation du quad et de mieux cerner la précision des mesures effectuées au théodolite ou en scooter de mer. Ces méthodes ont été très récemment appliquées aux levés réalisés durant la mission ECORS qui s'est déroulée à Biscarosse (juin 2007) et en mars-avril 2008 sur la plage du Truc-Vert.

\begin{abstract}
:
The METHYS group, regularly conduct topographic and bathymetric surveys on the Aquitanian coast. The quad equiped with a GPS is used; Additional measurements can be made with theodolite and jet-ski. The aim of this paper is to present detailed analysis on the different steps of computation (frequency of survey, grid of computation, interpolation methods...), in order to preserve centimeter accuracy on data collected. In particular, the use of quad is optimized. These methods were recently applied to bathymetric and topographic surveys during the mission ECORS that took place in March-April 2008 on the Aquitanian coast (Truc-Vert beach).
\end{abstract}

Mots-clés : Topographies - bathymétries - théodolite - transformation de Helmert - GPS

DOI: 10.5150/jngcgc.2008.057-P $\quad$ (disponible en ligne - http://www.paralia.fr - available online) 


\section{$1 \quad$ Introduction}

Depuis plusieurs années, l'équipe de recherche METHYS (Modélisation, Expérimentation et Télédétection en HYdrodynamique Sédimentaire) effectue 2 fois par mois des levés topographiques alternativement sur deux plages du littoral Atlantique (Desmazes, 2005) : la plage de Biscarrosse, fortement anthropisée, à environ $10 \mathrm{~km}$ au sud du bassin d'Arcachon et la plage du Truc Vert, à environ 12 $\mathrm{km}$ au nord du Bassin d'Arcachon; cette plage éloignée de toute infrastructure humaine est considérée comme évoluant naturellement (Gouriou, 2007).

Figure 1. Relevé complet effectué à Biscarrosse le 15 juin 2007 durant la campagne ECORS (SHOM/EPOC). La topographie est réalisée en quad (radiales rouges) et à pied (points bleus). La "laisse de basse mer" (en vert) s'étend sur plus de $6 \mathrm{~km}$. Les zones où un GPS étanche serait nécessaire, ont été mesurées au théodolite. De plus, au large, une bathymétrie sommaire est levée en scooter de mer. Les altitudes sont exprimées en mètres et rapportées au système IGN 69; les sigles (ADCP, PS...) correspondent à des instruments de mesure.

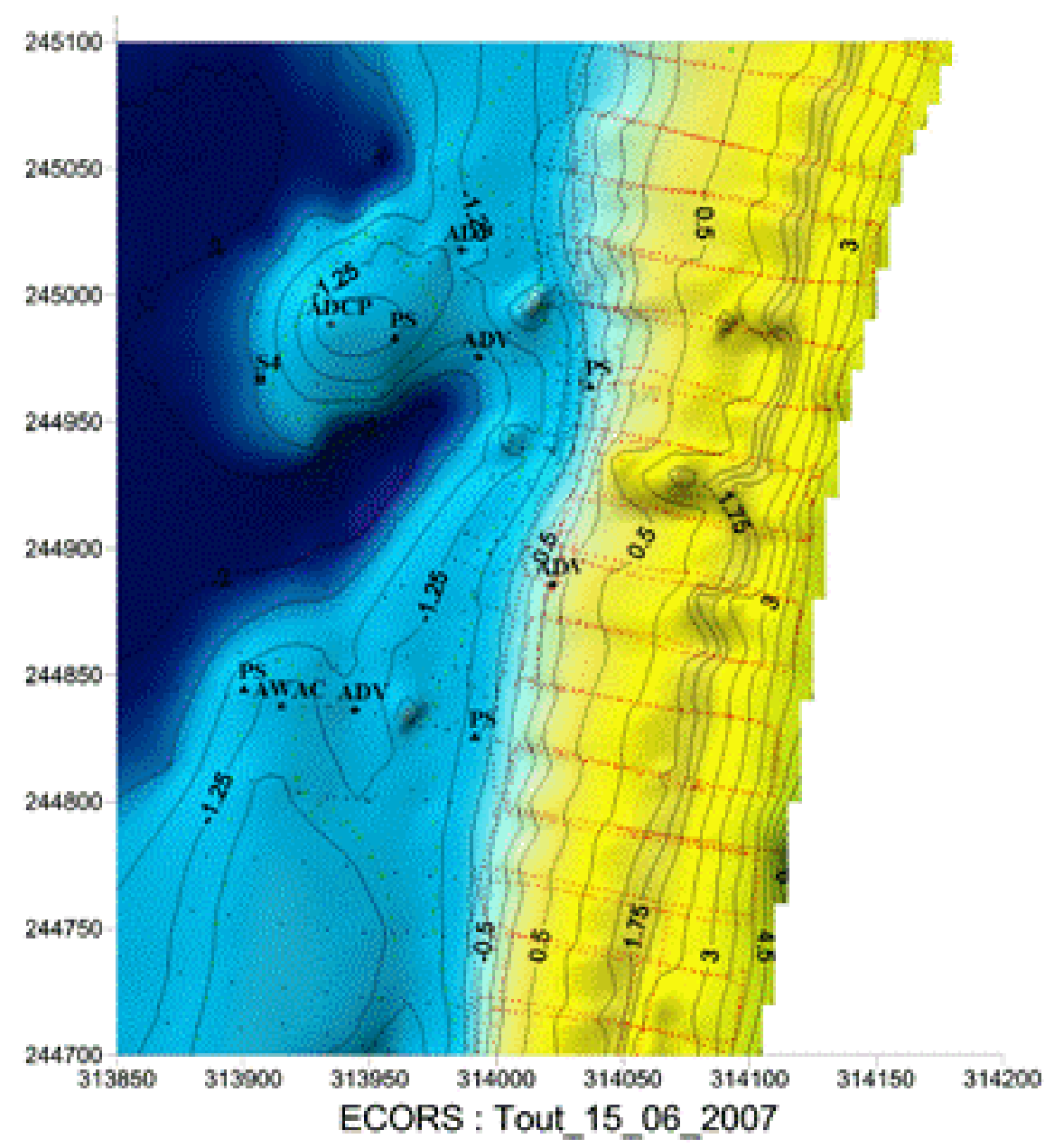


Le levé (figure 1) consiste à mesurer la topographie le long de profils appelés radiales, espacés de 10 à $25 \mathrm{~m}$ sur une distance longshore de $300 \mathrm{~m}$, depuis le haut de plage jusqu'à la ligne de rivage. Les levés sont effectués lors de marées basses de vives-eaux avec une acquisition mensuelle, depuis février 2006 à Biscarrosse et depuis 1997 au Truc Vert. Le trait de côte (laisse de basse mer) s'étendant sur 3 à $6 \mathrm{~km}$ est aussi réalisé.

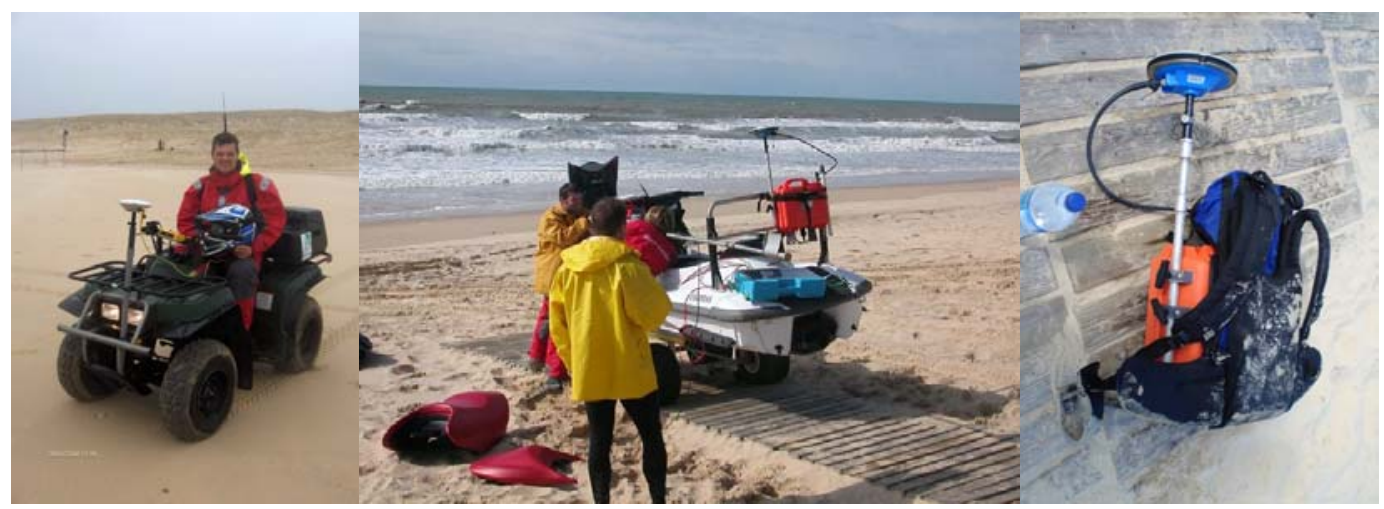

Figure 2. Levés GPS. De gauche à droite, Quad équipé d'une antenne ; préparation du scooter de mer avec l'antenne GPS ; le sondeur va être installé sur le coté) ; GPS étanche utilisé par l'équipe américaine durant la mission ECORS sur la plage du Truc-Vert en mars-avril 2008.

Les levés ont été effectués au théodolite, GPS, puis GPS cinématique depuis 2003, à pied de septembre 2003 à janvier 2007 ; en quad depuis janvier 2007. De plus, quand les conditions le permettent, dans les zones plus délicates ou inaccessibles à pied, des compléments de bathymétrie peuvent-être effectués au théodolite ou en scooter de mer (figure 1) équipé d'un sondeur et d'un GPS cinématique. Le matériel utilisé est un système de mesure centimétrique en temps réel, Trimble 5700. Ce dispositif comprend deux récepteurs, le mobile et la base. Une correction calculée par la base est apportée aux mesures par communication radio à $450 \mathrm{MHz}$. Le constructeur annonce pour le mobile, une précision horizontale de $\pm 1 \mathrm{~cm}$ et une précision verticale de $\pm 2 \mathrm{~cm}$. La base a été calibrée à partir des 2 points IGN les plus proches, situés à environ $20 \mathrm{~km}$ de distance de la plage. Récemment, la comparaison que nous avons réalisée avec les antennes du réseau RGP (réseau de stations GPS permanentes enregistrant les données provenant de satellites; l'IGN gère ces données provenant de 162 stations, qui sont mises à disposition des utilisateurs sur un site ftp public) a donné des résultats équivalents à quelques centimètres près. Des points de contrôle sont mesurés lors de chaque relevé; il s'agit de clous installés sur des invariants (poteau en bois, balise ONF, ouvrages bétonnés...) dont la comparaison régulière de la position nous assure un suivi de la précision des mesures. Des travaux 
semblables aux nôtres avec une fréquence moins élevée, ont été développés en Normandie sous la direction de F. Levoy (Stépanian, 2002 ; Anthony et al, 2005). Ces travaux ont débuté en 1991, avec des GPS transportés en quad ; une méthode originale (projet CLAREC) est en cours de développement avec l'utilisation d'un Laser à Balayage Latéral Aéroporté (LBLA) appelé également Lidar topographique. Cet équipement permet de réaliser une topographie fine du relief côtier sur de grandes surfaces.

\section{$2 \quad$ L'optimisation du QUAD}

\subsection{Le levé de référence}

Il s'agissait d'estimer les incertitudes liées au fonctionnement du quad: intervalle de mesure, vitesse du quad, espacement des radiales... Les données de référence utilisées pour optimiser le quad, ont été réalisées le 6 juin 2006 avec une maille d'acquisition de l'ordre de $2 \mathrm{~m}$, dont les caractéristiques sont données dans la figure 3.

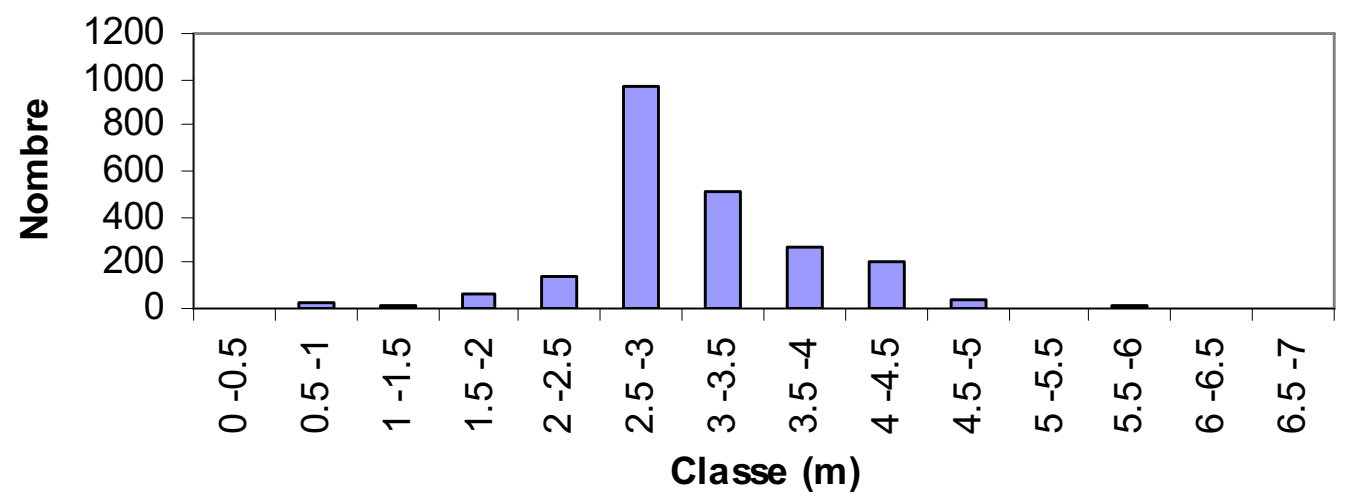

Figure 3. Histogramme des distances moyennes entre les points de mesure. Les données sont rassemblées par classes de $50 \mathrm{~cm}$.

On voit donc que ces distances moyennes sont de l'ordre de 2,5 m. Le jour de l'acquisition, le GPS enregistrait une donnée tous les $2 \mathrm{~m}$ ou toutes les deux secondes : c'est-à-dire que si au bout de 2 secondes, le quad n'a pas parcouru $2 \mathrm{~m}$, il acquiert quand même une valeur. Le quad soulève donc un problème par rapport aux relevés effectués à pied: si sur la plage se trouve une rupture de pente importante à prendre en compte comme une berme par exemple, son sommet n'est pas forcement enregistré. Nous avons donc réalisé de nombreux tests afin de chiffrer les différents biais d'origines variées : écartement des radiales, maille de calcul, méthode d'interpolation... 


\subsection{Ecartement des radiales}

La procédure utilisée pour évaluer l'importance de l'écartement des radiales est la suivante.

- Avec toutes les données, on reconstitue une surface $S$ interpolée avec une grille de $100 \times 100=10000$ points par exemple.

- Avec ces mêmes données, nous simulons des radiales d'espacements différents en réduisant à une seule, les données collectées dans un rectangle de cotés $\Delta x$ et $\Delta y$. Par exemple, pour simuler des radiales de $25 \mathrm{~m}$ (les radiales sont crosshore, parallèles à l'axe $x$ ), on prendra $\Delta x=1 \mathrm{~m}$ et $\Delta y=25 \mathrm{~m}$.

- A l'aide cette grille restreinte, on reconstitue la surface S' par interpolation avec le même pas et la même méthode que précédemment.

- Nous avons ainsi 2 surfaces reconstituées, l'une de référence (S), l'autre simplifiée ( $\left.\mathrm{S}^{\prime}\right)$. On effectue les différences d'altitudes et on trace l'histogramme de ces différences.

L'histogramme (figure 4) a été obtenu avec $\Delta x=1 \mathrm{~m}$ et $\Delta y=5 \mathrm{~m}$. L'histogramme montre que les écarts d'altitude ne dépassent pas $10 \mathrm{~cm}$. Si on utilise $\Delta x=1 \mathrm{~m}$ et $\Delta y=15 \mathrm{~m}$, on constate que certaines valeurs dépassent $10 \mathrm{~cm}$. Avec $\Delta x=1 \mathrm{~m}$ et $\Delta y=20 \mathrm{~m}$, certaines différences atteignent $20 \mathrm{~cm}$. En conclusion, les radiales du quad ne doivent pas excéder $10 \mathrm{~m}$ si 1 'on veut maintenir une cohérence meilleure que $10 \mathrm{~cm}$.

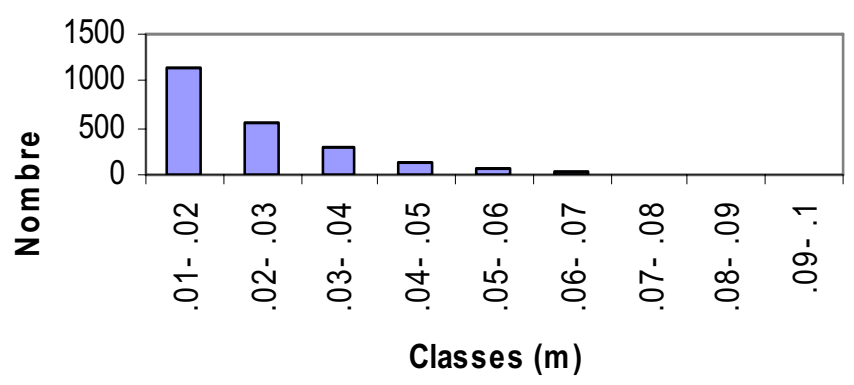

Figure 4. Histogramme des écarts entre la surface de référence et la reconstitution de la surface avec des radiales espacées de $5 \mathrm{~m}$. On peut noter que cet écart dépasse exceptionnellement $10 \mathrm{~cm}$. Les données sont rassemblées par classes de $50 \mathrm{~cm}$.

\subsection{Vérification des résultats sur une zone comportant un relief}

La maille très fine du 6 juin 2006 correspond à un profil de plage lisse, c'est-àdire, sans relief marquant. On peut alors se demander si on obtiendrait les mêmes résultats avec un profil de plage moins régulier. Le 7 avril 2007, après les grandes marées d'équinoxes, la plage du Truc Vert présentant des reliefs marqués, nous 
effectué un relevé avec une maille très fine, sur une étendue $62 \mathrm{~m}$ x $25 \mathrm{~m}$ sur une berme en croissant. Avec $\Delta x=1 \mathrm{~m}$ et $\Delta y=5 \mathrm{~m}$, on obtient l'histogramme de la figure 5 .

Si on désire une incertitude d'altitude inférieure à $10 \mathrm{~cm}$, l'espacement entre les radiales, doit être de l'ordre de $5 \mathrm{~m}$. Cela semble très contraignant, mais comme nous l'avons vérifié, la plupart des grandes différences d'altitudes sont dues à des effets de bords, là où l'interpolation des données est délicate.

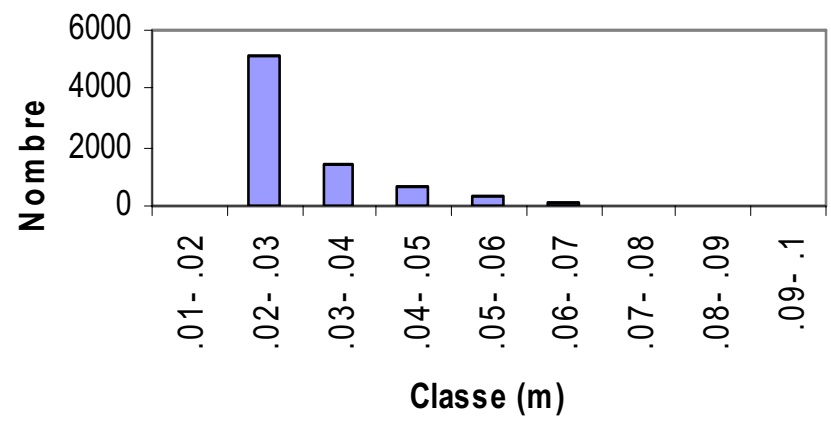

Figure 5. Histogramme des différences d'altitude pour le levé de la berme. Les données sont rassemblées par classes de $50 \mathrm{~cm}$.

\section{Test sur le pas de la maille}

Un critère que nous allons utiliser pour comparer les différentes méthodes de traitements est le calcul du volume de sable contenu dans le levé topographique. La taille de la maille de calcul est définie comme l'écart entre 2 points de calcul successifs : généralement on utilise un nombre de points identique dans les 2 directions.

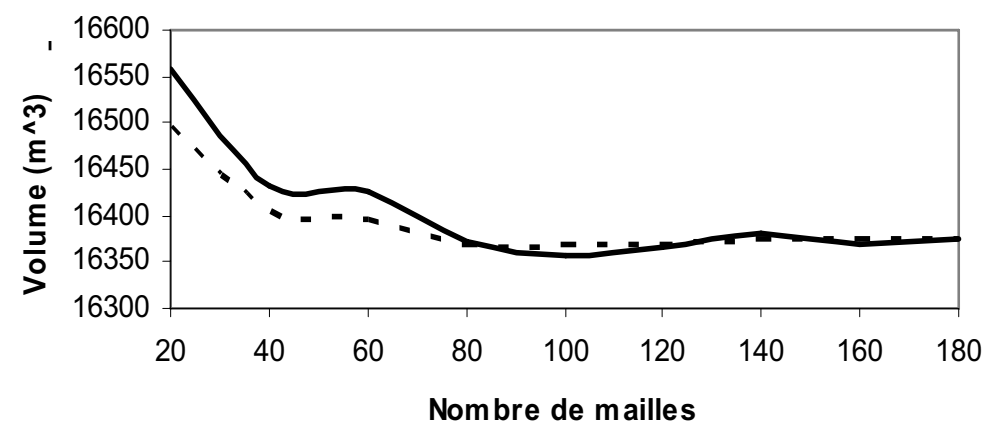

Figure 6. Evolution du volume en fonction du nombre de mailles de calcul. Plusieurs méthodes d'intégration sont superposées (Simpson, trapèzes...) et on voit, nettement, que toutes les méthodes convergent dès qu'on utilise environ 100 mailles, c'est-à-dire des pas de quelques mètres. On peut constater qu'au-delà de cette résolution, les écarts entre les différentes méthodes, sont inférieurs à $10 \mathrm{~m}^{3}$ pour un volume global de $16500 \mathrm{~m}^{3}$ 
Les tests que nous avons effectués sur le secteur couvrant environ $100 \mathrm{~m} \mathrm{x}$ $200 \mathrm{~m}$, vont de 20x20 à 180x180 points, ce qui correspond à une maille variant de $5 \mathrm{~m}$ à $1 \mathrm{~m}$. Ces différents maillages ont été choisis après une longue série de tests. La méthode d'interpolation est soit la méthode d'interpolation triangulaire, soit la méthode de Kriging implantée dans le logiciel Surfer que nous utilisons pour dépouiller des données. Nous ne présentons pas ici les nombreux tests réalisés sur le choix de la méthode d'interpolation et d'après nos premiers tests les méthodes «douces» sont préférables aux méthodes trop élaborées qui créent de l'information entre les points de mesure. D'autre part, le calcul de volume est effectué, avec des méthodes de quadratures diverses (Simpson, trapèzes...) : les écarts ne sont pas importants et par exemple la méthode des trapèzes est tout à fait adaptée. Finalement, on peut constater (figure 6) que toutes ces méthodes convergent avec un nombre de mailles de l'ordre de 100, soit des pas de l'ordre de 2 à $5 \mathrm{~m}$.

\section{Bathymétrie en scooter de mer}

La figure 7 présente des données levées en scooter de mer (pleine-mer) sur la plage du Truc-Vert le 19 mars 2008. Le Sondeur (PA500 à simple faisceau) effectue 10 mesures par seconde; le GPS donne 2 mesures par seconde. Dans la zone de recouvrement entres ces données et les données collectées à marée basse en quad, nous recherchons les points proches, c'est-à-dire dans un rayon de 50 $\mathrm{cm}$. Il y en environ 200, ce qui nous permet de déterminer par le calcul de la moyenne, le décalage du levé bathymétrique par rapport aux données GPS dont les altitudes sont en IGN69.

Figure 7. Bathymétrie effectuée le 19 mars 2008 sur la plage $d u$ Truc-Vert, en scooter des mers

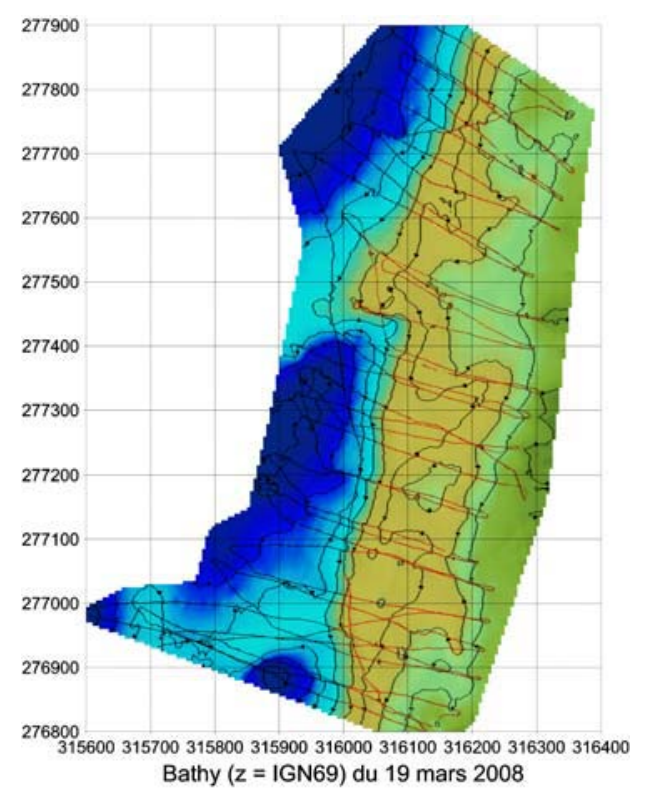


On constate (figure 8) que les données du sondeur sont en moyenne décalées de $1.10 \mathrm{~m}$ par rapport à l'IGN69. Par contre l'écart type entre les 2 jeux de données atteint $70 \mathrm{~cm}$, ce qui dénote la difficulté de ce type de mesures. Ceci est lié à 2 raisons, l'état très agité de la mer durant la mission ECORS (la hauteur significative de la houle, au large, est rarement descendue en dessous des $2 \mathrm{~m}$; le 19 mars elle était de $1.2 \mathrm{~m}$ ) et l'absence de mesures d'attitude du scooter, qui ne permet pas de connaitre l'orientation du sondeur. Par contre en supprimant les quelques points litigieux, l'écart-type reste de l'ordre de $40 \mathrm{~cm}$. Dans de meilleures conditions météorologiques, comme celles qui se sont améliorées en fin de mission, cet écart est descendu en dessous de $20 \mathrm{~cm}$. On peut comparer cette valeur à l'écart-type de $7 \mathrm{~cm}$ existant entre nos mesures topographiques et les levés effectués par le SHOM, durant la même mission, par très beau temps à bord des petites vedettes.

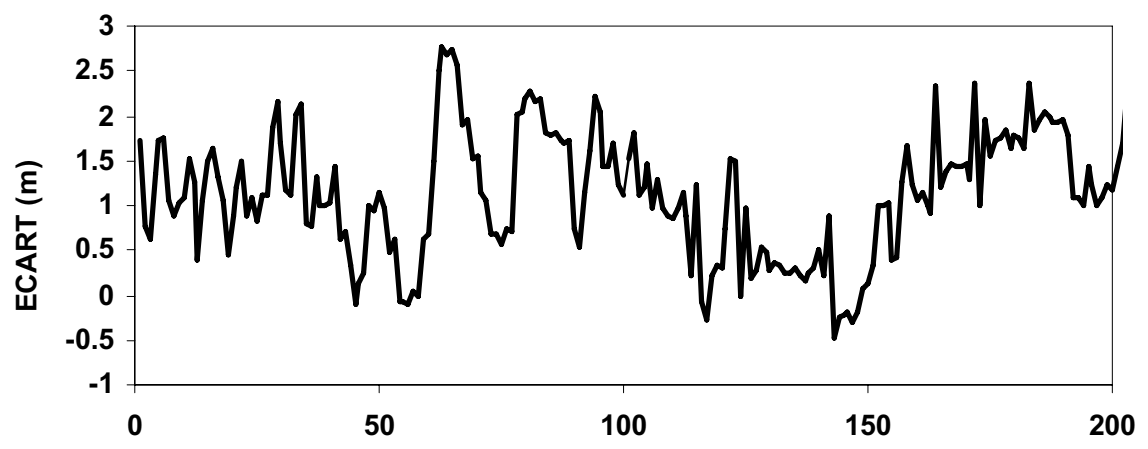

Figure 8. Ecart entre les « altitudes 》 des points communs. En moyenne les mesures du sondeur sont décalées de 1,10 m par rapport au 0 IGN.

\section{Transformation des données obtenues au théodolite}

Comme nous l'avons signalé, le théodolite intervient dans les conditions où le GPS ne peut être utilisé. De manière générale, on veut passer des coordonnées du théodolite $\left(\mathrm{O}^{\prime}, \mathrm{x}, \mathrm{y}, \mathrm{z}\right)$ à des coordonnées Lambert III $(\mathrm{O}, \mathrm{X}, \mathrm{Y}, \mathrm{Z})$ où $\mathrm{Z}$ est l'altitude IGN69. Dans le cadre de la transformation de Helmert à 4 paramètres (Milles S. et Lagofun J., 2007), on écrit $X=m x-n y+T_{x}, Y=n x-m y+T_{y}, Z=z+T_{z}$.

On fait l'hypothèse que la mise en station du théodolite est parfaite et que le plan de référence est parallèle au plan tangent au géoïde. La transformation se compose de 3 translations, d'une rotation et d'une homothétie. L'angle $\phi$ et le facteur $k$ sont donnés par $m=k \cos \phi, n=k \sin \phi, k=\sqrt{m^{2}+n^{2}}, \tan \phi=n / m$. Avec $N$ points de calage, le système s'écrit sous forme matricielle suivante ; la solution en termes de moindres carrés est $B^{T} A=B^{T} B P \Rightarrow P=\left(B^{T} B\right)^{-1} B^{T} A$. 


$$
\left(\begin{array}{c}
X_{1} \\
Y_{1} \\
X_{2} \\
Y_{2} \\
\cdots \\
X_{N} \\
Y_{N}
\end{array}\right)=\left(\begin{array}{cccc}
1 & 0 & x_{1} & -y_{1} \\
0 & 1 & y_{1} & x_{1} \\
1 & 0 & x_{2} & -y_{2} \\
0 & 1 & y_{2} & x_{2} \\
& & & \\
1 & 0 & x_{N} & -y_{N} \\
0 & 1 & y_{N} & x_{N}
\end{array}\right)\left(\begin{array}{c}
T_{x} \\
T_{y} \\
m \\
n
\end{array}\right) \quad A=B P
$$

La translation en altitude se calcule simplement en faisant la moyenne

$$
T_{z}=\frac{1}{N} \sum_{i=1}^{N}\left(Z_{i}-z_{i}\right)
$$

La transformation de Helmert à 7 paramètres se compose de 3 rotations, de 3 translations et d'une homothétie. Elle s'écrit de manière générale :

$$
\begin{aligned}
& R_{X}=\left(\begin{array}{ccc}
1 & 0 & 0 \\
0 & \cos \omega & \sin \omega \\
0 & -\sin \omega & \cos \omega
\end{array}\right) \quad R_{Y}=\left(\begin{array}{ccc}
\cos \theta & 0 & -\sin \theta \\
0 & 1 & 0 \\
\sin \theta & 0 & \cos \theta
\end{array}\right) \quad R_{Z}=\left(\begin{array}{ccc}
\cos \phi & \sin \phi & 0 \\
-\sin \phi & \cos \phi & 0 \\
0 & 0 & 1
\end{array}\right) \\
& F=\left(\begin{array}{ccc}
1+k & 0 & 0 \\
0 & 1+k & 0 \\
0 & 0 & 1+k
\end{array}\right) \quad T=\left(\begin{array}{c}
T_{x} \\
T_{y} \\
T_{z}
\end{array}\right) \quad M=\left(\begin{array}{c}
X \\
Y \\
Z
\end{array}\right) \quad m=\left(\begin{array}{c}
x \\
y \\
z
\end{array}\right) \quad M=F R_{X} R_{Y} R_{Z} m+T \\
& \text { (150 }
\end{aligned}
$$

Figure 9. Baïne levée au théodolite en juin 2007 à Biscarrosse. Les sigles représentent les instruments de mesures implantés durant la mission ECORS.

La détermination des 7 inconnues est résolue par une méthode de moindres carrés non-linéaires que nous avons développée avec les logiciels Maple et Matlab. L'intérêt de la comparaison est de chiffrer l'approximation liée à la réduction des paramètres de 7 à 4 . En particulier les angles $\theta$ et $\omega$ donnent une idée du défaut de mise en station du théodolite. Le jeu de données a été obtenu durant la campagne ECORS, à Biscarosse; c'est dans cette baïne (figure 9) que notre collègue Denis Michel a perdu la vie en juin 2007. Le tableau 1 compare les 
2 méthodes, et on peut noter la bonne qualité de l'approximation à 4 paramètres. En effet, les différents paramètres diffèrent de quelques $\mathrm{cm}$ pour les distances et de quelques centièmes de degrés pour les angles, tout à fait compatible avec la précision recherchée dans nos mesures.

\begin{tabular}{|c|c|c|}
\hline Paramètre & Helmert 4 & Helmert 7 \\
\hline $\mathrm{k}$ & 0,9983 & 0,9994 \\
\hline$\phi\left(^{\circ}\right)$ & 53,059 & 53,112 \\
\hline$\omega$ et $\theta\left(^{\circ}\right)$ & & 0,013 et 0,056 \\
\hline $\mathrm{T}_{\mathrm{x}}(\mathrm{m})$ & 314041,52 & 314041,58 \\
\hline $\mathrm{T}_{\mathrm{y}}(\mathrm{m})$ & 244922,72 & 244922,79 \\
\hline $\mathrm{T}_{\mathrm{z}}(\mathrm{cm})$ & 25,0 & 19,5 \\
\hline
\end{tabular}

Tableau 1. Comparaison des valeurs des paramètres du passage des coordonnées du théodolite au système Lambert III avec les 2 méthodes de Helmert.

\section{Conclusion}

A travers cette étude, nous avons abouti à une combinaison cohérente sur l'ensemble de données collectées pour la restitution de topographies et de bathymétrie obtenues avec des moyens variés : GPS, théodolite, quad, scooter de mer. Cela nous a permis d'optimiser l'utilisation du quad et de mieux cerner le traitement mathématique des données, en particulier les paramètres d'interpolation. Le point faible est l'obtention de mesures bathymétriques en scooter des mers, qui même dans de bonnes conditions présente des erreurs de $20 \mathrm{~cm}$. Par contre, ce travail montre l'excellente restitution obtenue pour la transformation en Lambert III, des données obtenues au théodolite.

\section{$7 \quad$ Bibliographie}

Anthony E.J. , Levoy F. , Monfort O. and Degryse-Kulkarni C. , 2005. Shortterm intertidal bar mobility on a ridge-an drunnel beach, Merlimont, northern France. Earth Surf. Process. Landforms 30, 81-93

Desmazes F., 2005, Caractérisation des barres sableuses d'une plage de la côte aquitaine. Thèse de doctorat, Université de Bordeaux I.

Gouriou T., 2007. Evolution morphologique d'une plage sableuse. Master ENVOLH. Bordeaux I.

Milles S. et Lagofun J., 2007, Topographie et topométrie modernes. Tome 2. Eyrolles, Paris.

Stépanian A., 2002. Évolution morphodynamique d'une plage macrotidale à barres : Omaha Beach Normandie. Thèse, université de Caen-Basse Normandie. 